\title{
Vivir en Frontera. Movimientos Socio-religiosos EN Chiapas y Guatemala
}

\author{
Grabriela Robledo Hernández
}

Resumen: En el presente trabajo se discuten los paralelismos de los movimientos de disidencia religiosa indígena en Chiapas y Guatemala, territorios que aunque pertenecen a Estados nacionales diferentes, comparten además de una frontera, una tradición histórica y cultural de larga data. Un significado compartido por estos movimientos es la construcción de nuevos proyectos colectivos sustentados en la experiencia del sentimiento numinoso, que al mismo tiempo proporcionan un capital social de suma importancia para estos pueblos quienes atraviesan por períodos de profunda transformación.

Palabras clave: movimientos sociorreligiosos, frontera, conversiones religiosas.

Enviado a dictamen: 25 de enero de 2010

Aprobación: 15 de junio de 2010

Revisiones: 2

Gabriela Robledo Hernández, Doctora en Ciencias en Ecología y Desarrollo Sustentable, Profesora investigadora del CIESAS Sureste, San Cristóbal de Las Casas, Chiapas, México. Temas de especialización: Religión, migración, género, Correo electrónico: grobledo@ciesas.edu.mx.
Abstract: In this paper I underline the similarities between the religious movements breaking out from the catholic church among indigenous people in Chiapas and Guatemala. In spite of their belonging to two different National States, they share a common cultural tradition, now split by a border. An important shared meaning of these religious movements, is the reconstruction of communalism in a time of deep social change.

Keywords: religious movements, border, religious conversions

\section{Introducción}

$\mathrm{E}$ n este trabajo planteo algunas ideas básicas en torno al paralelismo entre los movimientos de conversión religiosa que se iniciaron tanto en Guatemala como en Chiapas hacia la segunda mitad del siglo XX. Una amplia bibliografía ha documentado tanto la emergencia como las características de estos movimientos disidentes del catolicismo, volcados hacia la formación de nuevas congregaciones religiosas - genéricamente llamadas evangélicas-que se han multiplicado vertiginosamente en las regiones de ambos territorios.

Mi interés en el tema se desprende de un trabajo de investigación que he desarrollado desde hace algunos 
años entre los tsotsiles del altiplano chiapaneco quienes durante las últimas décadas han abandonado su religiosidad tradicional para abrazar las nuevas opciones religiosas que hoy proliferan en la región. Una parte de ellos viven en la ciudad de San Cristóbal, una ciudad turística de raigambre colonial, principal centro urbano regional. Mi acercamiento a esta población me ha permitido observar los vínculos que unen a las nuevas congregaciones religiosas con sus pares guatemaltecas. Confieso mi ausencia de trabajo de campo entre la población guatemalteca, pero en cambio he recurrido a los trabajos que recientemente han publicado un grupo de antropólogas norteamericanas y españolas interesadas en el tema.

La emergencia de una multitud de iglesias salvacionistas cristianas o paracristianas en el área, quienes a pesar de su origen externo se han "nativizado", me ha llevado a considerarlas como la expresión de un movimiento socio-religioso, concepto que ha sido utilizado por Barabas (2002) para describir los movimientos sociales de naturaleza religiosa con los que los pueblos indígenas de México históricamente han resistido y cuestionado el poder colonial, proponiendo nuevas utopías.

Sostengo que para los pueblos indígenas de Chiapas y Guatemala estos movimientos religiosos representan ensayos en la construcción de una nueva comunalidad, de la creación de nuevas formas de sociabilidad que llevan a la actualización de las identidades y a la reelaboración de las representaciones tanto colectivas como personales de los actores. El simbolismo religioso de estas expresiones permite la emergencia de un communitas que renueva la experiencia colectiva y legitima el reconocimiento mutuo de sus integrantes con base en un orden sagrado. La comunidad religiosa resulta entonces un espacio privilegiado para expresar la vitalidad y resurgimiento de un proyecto colectivo que ha sido amenazado por los súbitos cambios sociales que se han producido en estas sociedades.

\section{1.- Fronteras comunes, procesos paralelos}

Una característica de la región fronteriza ChiapasGuatemala es que los pueblos que en ella habitan comparten un legado histórico común. Además de ser el territorio donde han florecido los pueblos mayenses desde la antigüedad, ambas provincias formaban parte de la Capitanía General de Guatemala durante el período colonial, hasta que las dos alcaldías mayores de Las Chiapas se anexaron a México en 1824. Dieciocho años después, El Soconusco se integraría al Estado mexicano como parte de la entidad chiapaneca. El establecimiento de los límites entre México y Guatemala se formalizó hasta finales del siglo XIX (entre 1882 y 1894), aunque los intercambios comerciales, personales y familiares entre las poblaciones locales asentadas a ambos lados de la frontera continuaron intensamente.

La intensidad y permanencia de estos vínculos han significado un foco rojo para los intereses del Estado mexicano, quien en diversos momentos y a través de diferentes iniciativas ha emprendido políticas de mexicanización de la frontera. Así, durante el Porfiriato se alentó la colonización de la región de la Sierra por parte de campesinos guatemaltecos que más tarde se naturalizarían mexicanos. Posteriormente, durante el régimen cardenista, con Victórico Grajales como gobernador de Chiapas, se prohibiría la indumentaria y el uso de las lenguas nativas que se consideraban guatemaltecas en toda la región fronteriza (Hernández, 2001).

A pesar de estas acciones emprendidas por el Estado mexicano para definir los límites de su frontera septentrional, las poblaciones locales que en ella residen, resisten estas políticas negociando límites e identidades dentro de su territorio, construyendo las fronteras "vividas y sentidas" por ellos mismos, en tanto actores sociales (Wilson \& Donan, 1998). Las prácticas y creencias religiosas son parte de esta negociación y de la construcción de convergencias culturales 
entre pueblos hermanados históricamente, como lo acontecido con los movimientos de disidencia religiosa en Chiapas y Guatemala.

Así, en la zona fronteriza de la Sierra, el contacto con misioneros presbiterianos guatemaltecos dio origen a un movimiento religioso popular que se institucionalizó hasta 1920 con la fundación de la Iglesia Presbiteriana Espíritu Santo en el municipio de Mazapa de Madero. En esa región el presbiterianismo que defendía el uso de la lengua mam, en oposición a las campañas gubernamentales de imposición del castellano, se vio ligado a la etnicidad india. Años más tarde, un grupo de esta región, ya convertido a los Testigos de Jehová se trasladaría a la Selva para fundar un ejido (Hernández, 2005).

De manera paralela, el trabajo misionero del protestantismo guatemalteco se difundiría hacia Tapachula y la región del Soconusco de manera tímida y lenta hasta comprender a la población de las fincas cafetaleras (Rivera, 2003: 94). Aquí se produjo un encuentro entre indígenas guatemaltecos y tsotsiles y tseltales de la región del altiplano que históricamente desde finales del siglo XIX, primero de manera forzada y luego voluntariamente, iban año con año al corte del aromático. Testimonios de indígenas del altiplano señalan la importancia de este encuentro para la difusión del protestantismo en el altiplano chiapaneco.

Hacia la década de 1980, la región fronteriza de Chiapas se convirtió súbitamente en un área estratégica para la seguridad nacional ante los conflictos armados en Nicaragua, El Salvador y Guatemala (Ángeles, 2002). Fue una época en la que miles de campesinos indígenas, procedentes del occidente guatemalteco llegaron a la región fronteriza del territorio chiapaneco en calidad de refugiados, huyendo de una política contrainsurgente de tierra arrasada emprendida por los regímenes militares de Guatemala (Castillo, 2003).

El refugio permitió la convivencia de campesinos mexicanos y guatemaltecos por más de diez años. Para fines de la década de los 80, la Comisión Mexicana de
Ayuda a Refugiados (COMAR) reportaba la existencia de 119 núcleos de población refugiada con 22,800 habitantes en 6 municipios fronterizos chiapanecos (Las Margaritas, La Trinitaria, Frontera Comalapa, Bella Vista, La Independencia y Amatenango de la Frontera). Los campamentos de refugiados se establecieron en tierras ejidales o de propiedad privada, donde los guatemaltecos trabajaban como jornaleros con bajos sueldos, subarrendando terreno o en calidad de mozoscolonos. Con estas modalidades lograban usufructuar un pedazo de tierra para cultivar la milpa.

La presencia de la población refugiada en la franja fronteriza dinamizó el desarrollo de las políticas públicas hacia la hasta entonces desatendida población chiapaneca de la frontera. Hacia 1982 se inició la construcción de la carretera fronteriza del sur que une a través de $422 \mathrm{~km}$. a las ciudades de Comitán y Palenque, y rodea la frontera entre México y Guatemala, considerada zona de seguridad nacional. Esta vía fue inaugurada en el 2000 por el presidente Ernesto Zedillo. Pero, además, con la llegada de los refugiados también se empezó a desarrollar el proselitismo de los grupos protestantes en la franja fronteriza. Así, para fines de 1982, la Iglesia Presbiteriana formó un Comité de Auxilio a Refugiados con la ayuda de la Iglesia Nacional Presbiteriana, la Iglesia Reformada en América, la Iglesia Cristiana Reformada, La Iglesia Presbiteriana Unida en Estados Unidos y la Misión Centroamericana (Hernández, 1992). Este comité dirigía sus acciones en tres líneas: salubridad, alimentación y pastoral. Aunque la ayuda material en cuanto a salubridad y alimentación duró sólo algunos años, una política "pastoral de consolación" se extendió por más tiempo tanto entre la población guatemalteca como mexicana. Esta labor se refleja en el crecimiento de la afiliación no católica de la población de la región fronteriza, que en 1970 significaba sólo 1.6\%, cifra que aumentó a 8.21\% en 1980, a 12.92\% en 1990 y a $22.73 \%$ en el 2000 .

El trabajo misionero se vio ampliamente reforzado por radiodifusoras bilingües manejadas por los grupos 
protestantes que desde territorio guatemalteco eran escuchadas en la región. A principios de los 90 se escuchaba Radio Maya en kanjobal y la Voz de los Cuchumatanes en mam (Hernández, 1992).

Es necesario tener presente que Guatemala es considerado el país con la mayor proporción de población protestante en América Latina. Aunque el protestantismo se hizo presente en este país desde finales del siglo XIX con la emergencia de los regímenes liberales en Guatemala, no fue sino hasta la década de 1930 que empezó a florecer como resultado de un modernizante trabajo misionero de iglesias norteamericanas. Bajo esta influencia las comunidades indias del occidente guatemalteco empezaron a experimentar una profunda mutación religiosa, que acompañaba a un debilitamiento de la comunidad "tradicional", cuestionada por el abusivo y arbitrario poder que tenían entonces los zahorines (chamanes). En esta coyuntura, a las misiones protestantes se une un movimiento de reconquista de la Iglesia católica, en la forma de una Sociedad de Propagación de la fe que a partir de 1945 se transforma en Acción Católica (Le Bot, 1995).

A pesar de este origen externo, no tardaron en surgir Iglesias independientes entre los indígenas del altiplano guatemalteco, tales como la Iglesia Presbiteriana independiente, la Iglesia Nazarena Independiente, el grupo Príncipe de Paz y Ministerios Elim.

Hoy día los vínculos entre los grupos protestantes de los pueblos mayas cruzan las fronteras nacionales. Así por ejemplo Ministerios Elim es una iglesia pentecostal fundada en 1963 por Othoniel Ríos Paredes en la ciudad de Guatemala. Para 1974 la iglesia se convierte en Misión Cristiana Elim, expandiéndose a diversos países, entre ellos México. ${ }^{2}$ Actualmente Ministerios Elim tiene una importante presencia en Chiapas con 50 templos distribuidos en 14 municipios, la mayor parte de los cuales se ubican en municipios indígenas del altiplano: San Juan Chamula, Chenalhó, El Bosque, Simojovel, Amatenango del Valle, Zinacantán, Pantelhó, Oxchuc,
Tenejapa, Aldama, Chalchihuitán, Cintalapa, Teopisca y San Cristóbal. Cancino (2006) reporta la presencia de la iglesia Ministerios Elim "El Divino Salvador" MIEL entre los expulsados chamulas que habitan en la ciudad de San Cristóbal, quienes reconocen como jefe de su iglesia al apóstol Gaspar Zapalú, quien reside en Guatemala.

Los vínculos entre evangélicos guatemaltecos y chiapanecos se manifiestan de diversas maneras. La presencia de carismáticos pastores guatemaltecos en las grandes campañas protestantes que se realizan en el altiplano es de una notable atracción. Las radiodifusoras cristianas que transmiten desde territorio guatemalteco gozan de gran audiencia en la región fronteriza y en el altiplano chiapaneco y sirvieron de inspiración para la fundación de varias radios indígenas bilingües que transmiten desde la propia ciudad de San Cristóbal de Las Casas y que han surgido de manera ilegal en los últimos años.

La música es otro vínculo importante. Son muy populares las grabaciones de los grupos musicales cristianos tanto de origen guatemalteco como chiapaneco que cubren una amplia variedad de géneros: bolero, ranchero, música de banda, rap, rock, reagetton, etcétera, que consume la población cristiana e indígena de Chiapas. Los sitios de Internet de algunas de estas iglesias, como la de Ministerios Elim, también son centros de distribución de este tipo de música.

\section{2.- La modernización tardía y el proceso de secularización en Chiapas y Guatemala}

La otra idea que guia mi reflexión es que, tanto en Chiapas como en Guatemala, la conversión religiosa siguió a una modernización tardía y un concomitante proceso de secularización.

En Guatemala, el proceso masivo de conversión al protestantismo arranca en las primeras décadas del siglo XX. En Quezaltenango, el éxito de los presbiterianos se asocia a los efectos disruptivos del boom del café, la 
construcción de carreteras y el reclutamiento militar que produjeron transformaciones económicas y sociales en las comunidades indígenas del área (Green, 1993: 168). A principios de los 50 se inicia un proceso de modernización parcial y tardía en el país. ${ }^{3}$ Aunque los primeros contingentes de la migración ruralurbana hacia la ciudad de Guatemala procedían del oriente no indígena del país, en la década de los 70 la población indígena empieza a establecerse en la capital guatemalteca. El terremoto de 1976, que devastó el altipano guatemalteco, aunado a las campañas militares de tierra arrasada en esa región indígena mantienen los flujos hacia la ciudad de Guatemala hasta finales de la década de los 80 (Camus, 2002).

Chiapas, por su parte, anexada al naciente Estado mexicano en los albores del siglo XIX, se mantuvo como una sociedad conservadora donde la finca y la comunidad rural vivían en una especie de simbiosis. En algunas regiones como la del altiplano, el siglo XIX con su fiebre liberal y anticlerical fue aprovechado por las comunidades indígenas para librarse del yugo de párrocos y curas, construyendo en su lugar una especie de autonomía en materia de religiosidad local. Fue la época en que florecieron los sistemas de cargos, con el nombramiento de mayordomos, alféreces y una serie de funcionarios que tenían la finalidad de organizar y asegurar las celebraciones religiosas de carácter colectivo (Rus y Wasserstrom, 1980).

En aquellas otras regiones donde las comunidades se reprodujeron al interior de las fincas, como es el caso de los tojolabales, la vida religiosa colectiva, aunque de manera más simple, también se reprodujo en ese espacio autosuficiente.

Bien es sabido que los períodos históricos de la Revolución mexicana y la reforma agraria que transformaron el norte y el centro de México en las primeras décadas del siglo $\mathrm{XX}$, fueron periodos de reacomodamiento y ajuste de las élites regionales dominantes en tierra chiapaneca. Aquí, el reparto agrario se aplicó de manera parcial, selectiva y tardía, afectando sólo algunas áreas que correspondían a las tierras menos productivas del agro chiapaneco.

Así las cosas, la modernización de las relaciones sociales en la entidad a través del desarrollo de las relaciones capitalistas irrumpiría abruptamente hasta la segunda mitad del siglo XX cuando hacia la década de los 70 se emprendió una política nacional de modernización en la entidad. Se inició la construcción de las presas hidroeléctricas en los valles centrales, la explotación del petróleo en Tabasco y Chiapas y la emergencia de la industria turística en las ciudades del Caribe mexicano, lo que posibilitaría la incorporación de la abundante mano de obra de las región del altiplano chiapaneco a la industria de la construcción en estas zonas.

A partir de esta modernización tardía se empezó a producir una rápida e incierta transformación de la sociedad chiapaneca y de los diversos mundos que en ella habitan. En materia religiosa un concomitante proceso de secularización se presentó de manera abrupta y hasta violenta en las comunidades indígenas del altiplano. En ellas, las presiones centrífugas de diversos tipos sobre la comunidad agraria habrían de romper el tradicional mundo de seres que custodiaban los espacios colectivos, y al que se rendía culto a través de las celebraciones religiosas de carácter anual. El rito religioso, acto social básico a través del cual se construía un mundo comunitario con un sentido único y obligatorio para todos, se rompió. De manera paralela se rompieron los consensos políticos de la vida comunitaria y aparecieron las migraciones forzadas que obligaron al traslado de miles de familias fuera de sus municipios de origen a través de lo que se conoció como expulsiones religiosas (Robledo, 1997; Betancourt, 1997, Pérez, 1989).

Desde el altiplano se producirían corrientes migratorias hacia otras regiones del estado. Algunas de éstas irían a colonizar la selva, y bajo la influencia de una pastoral social católica renovada e inspirada en la teología de la liberación buscarían construir en ese territorio su tierra prometida. Otras se dirigieron a 
los Chimalapas donde competirían con otros colonos por un territorio rico en recursos, pero disputado entre Oaxaca y Chiapas. Los más se dirigieron a las ciudades, principalmente a San Cristóbal de Las Casas y sus alrededores, y a la ciudad de Tuxtla Gutiérrez, la capital del estado.

El movimiento zapatista vendría a polarizar aún más los grupos e intereses al interior de las comunidades, incrementándose con ello los desplazamientos masivos de carácter forzado de campesinos indígenas a las ciudades (Rebón, 2001). Así, San Cristóbal, lugar histórico de asentamiento de la población primero española y luego ladina o mestiza, hoy tiende a convertirse cada vez más en una ciudad mayoritariamente indígena. El levantamiento abrió una coyuntura para la apropiación de tierras por parte de las comunidades indígenas en varias regiones de Chiapas a través de las invasiones a predios particulares.

En materia religiosa, la presencia indígena en la ciudad ha traído consigo el florecimiento del pluralismo y la multiplicación de la oferta religiosa. Ésta incluye una amplia gama de doctrinas: desde la propuesta católica de la iglesia autóctona, la diversidad de grupos protestantes que incluye a presbiterianos, bautistas, pentecostales y neopentecostales; grupos no protestantes como Testigos de Jehová y mormones, hasta la presencia de dos comunidades musulmanas que se han desarrollado recientemente.

\section{3.- La recreación de la comunidad}

Si en el mundo rural tradicional dominaban las identidades parroquiales, donde la localidad y la familia extensa jugaban el papel primordial, en la ciudad predominan las interacciones entre familias de diversos municipios indígenas de la región que conviven en los asentamientos periféricos de la ciudad. En este nuevo mundo urbano, los grupos religiosos se han convertido en un nuevo espacio comunitario donde se renuevan las manifestaciones culturales propias y se construye un capital social de suma relevancia para enfrentar las precariedades tanto materiales como sociales que conlleva el traslado del campo a la ciudad. Es en el contexto urbano donde la religión se constituye en el eje a partir del cual se construyen elementos simbólicos que se integran a una matriz cultural propia para fortalecer su identidad cultural y étnica a partir de horizontes más amplios.

Las manifestaciones de la religiosidad indígena urbana se caracterizan por una especie de "tribalismo religioso", aludiendo a las "tribus" del mundo posmoderno a las que se refiere Maffesoli (2004). Se trata de pequeñas comunidades emocionales que permiten el reconocimiento mutuo, la expresión de un sentir en común, ligado a la proxémica, una manera de estar juntos, compartiendo un mismo territorio real y simbólico. En estos pequeños grupos, la ritualidad religiosa gira en torno a la expresión de los sentimientos que se expresan en un tiempo-espacio colectivo de una manera inmediata, sensible y afectiva. Un hombre tsotsil me explicó su identificación con una comunidad religiosa porque era "caliente", haciendo alusión a la experiencia emocional que experimentaba en ella.

La vida cotidiana es el espacio-tiempo para el "estar-juntos", por lo que la cultura local influye sobre el conjunto de las creencias, normas y valores de la comunidad de creyentes, creando un sistema de significaciones que permite el reconocimiento mutuo. El uso de la lengua nativa en el culto, la oración y el ayuno como vía de acercamiento a la divinidad, el uso de la indumentaria tradicional para asistir al culto son ejemplos del estilo de socialidad que caracteriza a estas congregaciones. Considero que estos dos ejes explican el éxito de las nuevas opciones religiosas: la expresión de las culturas locales y la dimensión emocional que subraya la experiencia afectiva de comunión colectiva.

En la dinámica religiosa aquí descrita hay una cierta "autonomización" de las comunidades religiosas, en ella se observa una separación entre creencia e 
identificación. Es decir, la pertenencia a una u otra congregación no es cuestión de elección doctrinal, sino de la sociabilidad que acompaña a una u a otra. La identificación religiosa en estas condiciones se vuelve un hecho efímero, móvil e inestable que se mueve a la par de un faccionalismo político y de la manera en que uno u otro grupo proporcionan un capital social efectivo para solucionar las carencias de la vida cotidiana. La identificación religiosa en estas circunstancias, más que un hecho individual, responde a una iniciativa grupal que involucra a miembros de la familia, paisanos o vecinos. Nos encontramos frente a una socialidad donde domina la interacción entre pequeños grupos que forman una comunidad en el mundo urbano.

En el caso guatemalteco algo similar parece estar ocurriendo. Ahí, la violencia de Estado golpeó brutalmente a los pueblos indígenas, por lo que los templos llenaron el vacío social dejado por la destrucción de comunidades y familias. Los trabajos de Cantón $(1998,1999)$ y Green (1993) subrayan el papel de las congregaciones religiosas donde se reconstruyen lazos de cooperación, confianza y comunalidad, a través de prácticas de ayuda y apoyo mutuo, al tiempo que se convierten en fuentes de reconocimiento y solidaridad intragrupal. Para muchas mujeres viudas del occidente guatemalteco afiliarse a las iglesias cristianas se ha convertido en una estrategia de sobrevivencia ante los profundos trastornos sociales por los que atraviesa su mundo.

\section{Reflexión final}

Aunque las fronteras internacionales son espacios de delimitación entre un "nosotros" y los "otros" que incluyen límites legales que separan y al mismo tiempo unen a los Estados nacionales, son también zonas intersticiales donde se presentan fenómenos de desplazamiento y desterritorialización que producen una identidad híbrida y contradictoria en los sujetos y las colectividades.
En el caso que aquí examinamos, a pesar de las diferencias socio estructurales de los Estados nacionales que contienen a los pueblos que habitan en Guatemala y Chiapas, encontramos profundas conexiones que se manifiestan en procesos paralelos de construcción de significados en períodos de intensa crisis y transformación. Las nuevas prácticas y creencias religiosas compartidas por los pueblos nativos de ambos territorios expresan los paralelismos de sentido de una experiencia religiosa que se alimenta de una matriz y un destino común.

En ambos casos se ensayan nuevos modelos de interacción a través del florecimiento de congregaciones religiosas de diversa denominación que responden a una búsqueda y renovación de una interacción de tipo communitas, caracterizada por la interacción de seres concretos, históricos y totales en un modelo de comunicación Yo-Tú, independiente del estatus y función que ocupan en la estructura social. Aunque este modelo de interacción está normado por las prescripciones de cada iglesia, el culto religioso permite crear un espacio espontáneo a partir de la experiencia del sentimiento de lo numinoso y lo sublime, propio de toda experiencia religiosa. A través de esta experiencia emocional que puede llegar a ser extática, se alcanza un profundo sentido de comunión, de compartir con los iguales: "hermanos y hermanas".

Este modelo de interacción, fluido y espontáneo se encuentra en tensión con la congregación, vista como parte de una estructura eclesiástica mayor sujeta a normas y controles por una autoridad externa y jerárquicamente superior. Es en este nivel de la comunidad, vista como estructura, donde tiene lugar la competencia por el liderazgo, que regularmente conduce al faccionalismo y a la división de una congregación para dar origen a otra nueva.

La existencia de estos proyectos alternativos de construcción colectiva cuestiona la noción de comunidad que hasta ahora había prevalecido en la visión de la antropología regional, donde ésta aparece 
como un cuerpo unívoco, armonioso y homogéneo. Las comunidades indígenas hoy en día son atravesadas por distintos proyectos societales que desde la sociedad dominante, nacional y global son reinterpretadas y reelaboradas por los actores. Junto con estas propuestas emergen el conflicto y la confrontación. Asistimos entonces a un reajuste de las dinámicas comunitarias en ambos territorios, donde poco a poco se va abriendo paso a la diversidad.

El estudio de la naturaleza y dinámica de los vínculos de los grupos religiosos guatemaltecos y chiapanecos es aún una agenda pendiente. No se ha analizado de manera suficiente y con profundidad la creación de comunidades religiosas que en ambos territorios construyen un entramado de significados compartidos, desafiando los límites de sus respectivos Estados nacionales. Para chiapanecos y guatemaltecos, esta religiosidad forma parte de su manera de vivir en frontera.

\section{Notas}

${ }^{1}$ Uno de estos testimonios es el del líder Domingo López Ángel, quien se convirtió al pentecostalismo cuando trabajaba en una finca del Soconusco.

${ }^{2}$ Paraconsultarmássobreelorigendeestegrupover:http:// groups.msn.com/MinisteriosELIMGuatemalaCentral. [Consultado el 27 de octubre de 2008].

${ }^{3}$ Camus (2002: 52) señala que la Revolución de Octubre de 1944 sienta las bases de un moderno desarrollo capitalista que se ve frenado por un Estado oligárquico que se mantiene de manera coercitiva a través del permanente ejercicio de la violencia.

\section{Bibliografía}

Barabas, Alicia (2002), Utopías indias. Movimientos sociorreligiosos en México, México: Instituto Nacional de Antropología e Historia/Plaza y Valdés, tercera edición.
Betancourt, Darío (1997), Bases regionales en la formación de comunas rurales-urbanas en San Cristóbal de Las Casas, Chiapas, Tuxtla Gutiérrez, Chiapas: Universidad Autónoma de Chiapas.

Camus, Manuela (2002), Ser indígena en ciudad de Guatemala, Guatemala: FLACSO.

Cancino, María del Socorro (2006), Religión y género: el caso de las chamulas expulsadas, Tesis de maestría en Ciencias Sociales con opción en estudios fronterizos, Universidad de Ciencias y Artes de Chiapas, Centro de Estudios Superiores de México y Centroamérica.

Cantón, Manuela (1998), Bautizados en fuego. Protestantes, discursos de conversión y política en Guatemala (1989-1993), La Antigua, Guatemala, Vermont, E.U.: Centro de Investigaciones Regionales de Mesoamérica/ Plumsock Mesoamerican Studies, South Woodstock.

Cantón, Manuela (1999), "La rebelión invisible. Mujeres latinoamericanas y conversión religiosa", en Pilar Sanchíz e Isabel Ma. Martínez (coords.), Mujeres latinoamericanas: entre el desarrollo y la superviviencia, Sevilla, España: Universidad Internacional de Andalucía.

Castillo, Manuel Ángel (2003), "The Mexico-Guatemala Border: New Controls on Transborder Migrations in View of Recent Integration Schemes", en Frontera Norte, vol. 15, núm. 29, enero-junio.

Green, Linda (1993), "Shifting Affiliations: Mayan Widows and Evangélicos in Guatemala”, en Virginia Garrard-Burnett y David Stoll (ed.), Rethinking Protestantism in Latin America, Philadelphia, USA.: Temple University Press.

Hernández, Castillo, Rosalva Aída (1992), "Los refugiados guatemaltecos y la dinámica fronteriza en Chiapas", en Graciela Freyermuth y Rosalva Aída Hernández (comp.) Una década de refugio en México. Los refugiados guatemaltecos y los derechos humanos, México: Ciesas/Instituto Chiapaneco de Cultura y Academia de los derechos humanos. 
Hernández, Castillo, Rosalva Aída (2005), "Protestantismo, identidad y poder entre los mayas", en Mario Humberto Ruz y Carlos Garma (ed.), Protestantismo en el mundo maya contemporáneo, México: UNAM/UAM

Le Bot, Yvon (1995), La guerra en tierras mayas. Comunidad, violencia y modernidad en Guatemala (1970-1992), México: F.C.E

Mafessoli, Michael (2004), El tiempo de las tribus. El ocaso del individualismo en las sociedades posmodernas, México: Siglo XXI.

Martin, David (1991), Tongues of fire. The Explosion of Protestantism in Latin America, Great Britain: Blackwell:

Pérez, María Isabel (1989), Expulsiones indígenas. Religión y Migración en tres municipios de Los Altos de Chiapas: Chenalhó, Larráinzar y Chamula, México: Claves Latinoamericanas.
Rebón, Julián (2001), Conflicto armado y desplazamiento de población. Chiapas 1994-1998, México: FLACSO/Miguel Ángel Porrúa.

Rivera, Carolina (2003), Dinámica del crecimiento evangélico en Chiapas. El caso del Valle de Pujiltic, Tesis de doctorado en Antropología, UNAM/IIA.

Roberts, Bryan (1968), "Protestant Groups and Doping with Urban Life in Guatemala City", en The American Journal of Sociology, vol. 73, no. 6, pp. 753-767.

Robledo, Gabriela, 1997, Disidencia y Religión. El caso de los expulsados de San Juan Chamula, Tuxtla Gutiérrez, Chiapas: UNACH.

Rus, Jan y R. Wasserstrom (1980), "Civil-Religious Hierarchies in Central Chiapas: a Critical Perspective", en American Ethnologist 7 (3), pp. 466-478.

Wilson, T. \& H. Donan (eds.) (1998), Border identities. Nation and State at International Frontiers, England: Cambridge University Press. 